\title{
Socially-Aware Recommender Systems
}

\author{
Nana Yaw \\ Asabere \\ Computer Science \\ Department, \\ School of Applied \\ Science and Arts, \\ Accra Polytechnic, \\ Accra, Ghana
}

\author{
Wisdom Kwawu \\ Torgby \\ Computer Science \\ Department, \\ School of Applied \\ Science and Arts, \\ Accra Polytechnic, \\ Accra, Ghana
}

\author{
Tonny Montana \\ Adegboyega \\ Computer Science \\ Department, \\ School of Applied \\ Science and Arts, \\ Accra Polytechnic, \\ Accra, Ghana
}

\author{
Dorothy Anima \\ Frempong \\ Computer Science \\ Department, School \\ of Applied Science \\ and Arts, \\ Accra Polytechnic, \\ Accra, Ghana
}

\begin{abstract}
In current social networks, users are characterized by social properties. Are these social properties relevant in a recommendation process? Can social properties such as social ties and degree centrality of users be applied to generate effective recommendations? In answering these questions, the authors draw pertinent literature and argue that indeed the incorporation of social properties in recommender systems is very significant for generating reliable recommendations for users. The authors envisage that innovative research in recommender systems should integrate the social properties of users in order to generate trustworthy and efficient recommendations.
\end{abstract}

\section{General Terms}

Social Properties, Recommender Systems

\section{Keywords}

Mobile Social Networks, Online Social Networks, Recommender Systems, Social Properties.

\section{INTRODUCTION}

In the past few years, social networks have been corroborated as exceptional communication tools that are used to represent and analyze data emerging from social interactions and activities of users. Globally, the thorough study of social networks can help both researchers and users understand the emergence of different types of phenomena. The amount of available social network data is growing day by day and this introduces many computational problems such as information overload for effective analysis of data [1], [2].

With the advent of mobile technology and devices, social networks are rapidly becoming mobile, which has introduced Mobile Social Networks (MSNs). Most OSNs regard their advancement into mobile as one of the key initiatives to their growth. Globally, many famous companies such as Yahoo and Google are currently making strategic efforts, strides and initiatives to integrate MSN technologies [2]. Through social network analysis, various social properties can be extracted or mined from OSNs as well as MSNs and applied to diverse recommender applications [2], [4].

Recommender Systems (RSs) are software applications that are relevant to social networks and attempt to reduce information overload by recommending items of interest to end users based on possible interests such as tourist sites/destinations and movies [5]. A novel application of MSNs target recommender systems. By tracking the behavior of users and mining the social and context information available in their mobile devices, recommender systems can generate effective recommendations on a variety of topics [2].
In social networks, the social properties of users, such as social ties, communities, friendship and centrality can be inferred, computed and used as additional incentives and motivations to recommender system entities. These social properties can be combined with recommender system entities such as context, items and user interests to improve the generation of social recommendations. This paper seeks to substantiate the importance, significance and necessity of modeling a recommendation process through integration of some social properties.

For example the computation of social ties between two users in a social network can help to determine the extent of their relationship, so that an effective recommendation can be generated for either of the users. Furthermore, degree centrality can be employed to compute the popularity of users in order to generate trustworthy recommendations, in scenarios where popularity is being considered for a recommendation. Additionally, community detection techniques can be utilized to determine the recommended communities that users belong to in a social network.

The rest of the paper is organized and structured as follows. Section 2 elaborates on recommender systems in social networks. Section 3 discusses modeling and evaluating the performance of socially-aware recommender systems. Finally, Section 4 concludes the paper.

\section{RECOMMENDER SYSTEMS IN SOCIAL NETWORKS}

In social networks, recommender systems demonstrate a function of producing a ranked list of items which might be of interest to a user based on his/her preferences. Recommender systems are designed for commercial activities (books, movies, TV shows etc.) as well as educational purposes (research articles, e-learning, m-learning etc.). There are two main traditional approaches that are used to design a recommender system for social networks, these are Collaborative Filtering (CF) and Content-Based Filtering (CBF).

\subsection{Traditional and Context-Aware Recommender Systems}

$\mathrm{CF}$ is one of the most successful recommendation approaches. $\mathrm{CF}$ computes the similarity between two users based on their rating scores and recommends an item which is highly rated by similar users. Unlike other approaches which are based on essential consumer and product characteristics, CF personifies consumers and products implicitly using their previous interactions. Researchers are advancing $\mathrm{CF}$ technologies in areas such as algorithm design, human-computer interaction design, and consumer incentive analysis. On the other hand, 
$\mathrm{CBF}$ recommenders suggest products/items to a user with a focus on the products/items the user preferred/liked in the past. Unlike CF, in CBF there is no comparison between the rating scores and similarity of users [5].

In order to generate relevant recommendations, a ContextAware Recommender System (CARS) does not only make use of user preferences and interests, but also exploits information about the specific contextual situation in which the recommended item will be consumed. The adaptation of recommendations to the user's current contextual situation requires an understanding of the relationship between user preferences and contextual conditions [6].

Table 1: Summary of Social Properties

\begin{tabular}{|c|c|}
\hline $\begin{array}{l}\text { Social } \\
\text { Properties }\end{array}$ & Description/Meaning \\
\hline $\begin{array}{l}\text { Betweenness } \\
\text { Centrality }\end{array}$ & $\begin{array}{l}\text { The extent to which a node/user lies on } \\
\text { the geodesic path that links other } \\
\text { nodes/users. }\end{array}$ \\
\hline $\begin{array}{l}\text { Closeness } \\
\text { Centrality }\end{array}$ & $\begin{array}{l}\text { The shortest path between a node/user } \\
\text { and other reachable nodes/users. } \\
\text { Closeness centrality is calculated through } \\
\text { the reciprocal of the mean geodesic } \\
\text { distance. }\end{array}$ \\
\hline $\begin{array}{l}\text { Degree } \\
\text { Centrality }\end{array}$ & $\begin{array}{l}\text { The number of direct ties that are linked } \\
\text { to a given node/user. }\end{array}$ \\
\hline Social Tie & $\begin{array}{l}\text { Significant social relationships that are } \\
\text { featured by the blend of intimacy, } \\
\text { emotional intensity, amount of time, } \\
\text { regularity and social homogeneity. }\end{array}$ \\
\hline $\begin{array}{l}\text { Social } \\
\text { Neighbors/ } \\
\text { Friendship }\end{array}$ & $\begin{array}{l}\text { The set of nodes/users with which a host } \\
\text { is related through specific social ties, } \\
\text { preferably strong. Also called friendship. }\end{array}$ \\
\hline Communities & $\begin{array}{l}\text { A combination or clustering of entities } \\
\text { that are closely related and linked to each } \\
\text { other, either through some easily } \\
\text { accessible entities that can act as } \\
\text { intermediates or by direct linkage. }\end{array}$ \\
\hline
\end{tabular}

\subsection{Socially-Aware Recommendation}

Social networking has become an actuality, and it generates tremendous amount of information that is sometimes difficult for users to process, especially from mobile phones [7]. There is therefore the need to filter and make accessible such information to users. Consequently, researchers are motivated to develop mobile recommender systems that exploit social network information [7]. Additionally, recommendation schemes and systems that are based on matchmaking in multiple MSNs, and can help users to find their potential friends without disclosing their private information are significant and necessary [8].

A model-based approach for recommendation in social networks can be executed by using matrix factorization

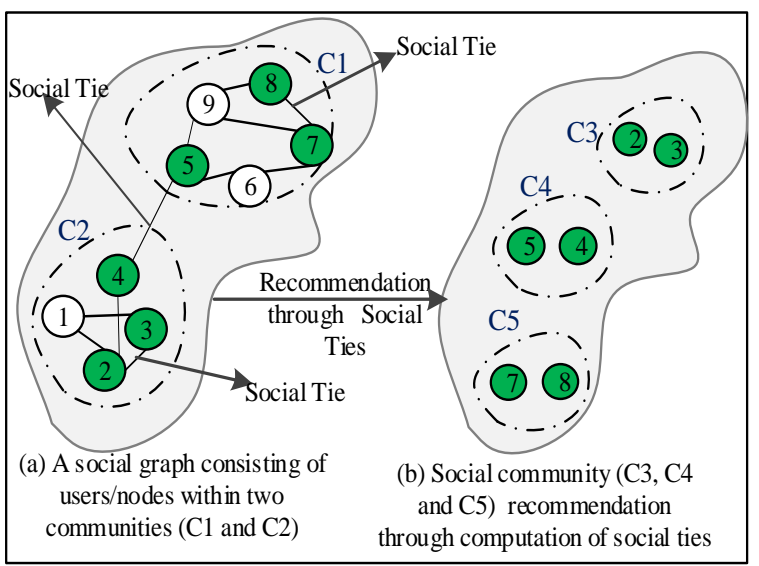

Fig. 1: Illustration of Recommendation Through Social Ties (a) A Social Graph Consisting of Users/Nodes Within two Communities (C1 and C2) (b) Social Community (C3, C4 and C5) Recommendation Through Computation of Social Ties

techniques [9]. Most people agree that there are new challenges of promoting socially-aware recommendation. Discovering the potential of the current immense and everincreasing information will foster a more efficient delivery of mobile services [10]. Furthermore, social recommenders can be based on graphs built on the basis of data mining components that extract knowledge about the relations and information exchanged by users [11]

\subsection{Integration of Social Properties in Recommender Systems}

It can be realized that the traditional recommender systems utilize two main entities i.e. items and user interests to generate recommendations. Subsequently, various contextual information and some socially-aware techniques such as matrix factorization, data mining and matchmaking schemes have also been modeled and developed by researchers as part of different recommendation processes. This section of the article elaborates on the possible scenarios of how some type of social properties can also be used as entities in sociallyaware recommendation. In particular, we discuss some social properties of MSNs such as social ties, centrality, communities and friendship.

\subsubsection{Social Ties and Tie Strength}

In a social network, a person's contacts is characterized by both strong and weak ties. Strong ties are more likely to be activated for information flow when compared to weak ties. Tie strength is a quantifiable property that characterizes the link between two nodes/users [12].

When incorporating tie strength indicators in recommender systems, some factors which should be considered include: intimacy, emotional intensity, amount of time, frequency and social homogeneity [2], [4]. For example, to compute the social tie between two nodes/users, $a$ and $b$ in a social network, it is pragmatic to use equation (1).

$$
\operatorname{SocTie}_{a, b}(t)=\left(\lambda_{a, b} \times d_{a, b}(t)\right) / T
$$

where $d_{a, b}(t)$ is the contact duration between the nodes/users, $a$ and $b$ in the time frame $T$ and $\lambda_{a, b}$ is their contact frequency (i.e. the number of times $a$ and $b$ have been in contact within the time frame $T$ ). A stronger social tie can successfully 
generate a reliable and an efficient recommendation for either of the nodes/users.

Figure 1 illustrates an example of generating a recommendation through the computation of social ties for different users/nodes in communities within a social graph. In Figure 1 (a), nodes/users that have social ties are directly linked to each other to form the social communities depicted in Figure 1 (b).

\subsubsection{Centrality}

In the fields of Graph Theory and Social Network Analysis, the term centrality is defined as the quantitative measure of the topological importance of a vertex within a graph. In a social graph, a central node/user, typically has a stronger ability to connect to other nodes/users in the same graph. Furthermore, the centrality of a node describes the social importance of its represented person in the social network [2], [4], [13].

Numerous centrality measures have been used for characterizing the social behaviour and connectedness of nodes within networks [13], [14]. As shown in Table 1, the centrality within a social graph can be defined in three ways, namely: degree centrality, betweenness centrality and closeness centrality.

Degree centrality is the simplest centrality measure and is defined as the number of direct contact (links) connected to a given node/user. A node/user with a high degree centrality is defined as a popular node with a large number of possible direct contacts and thus a good candidate for recommender systems in terms of user popularity.

For instance, the popularity of a presenter at a smart conference can be computed through his/her degree centrality at the conference community. Consequently, a recommendation can be generated for participants of the conference as a result of the computed degree centrality (popularity) of the presenter(s).

Degree centrality for a given node $c$, where $a(c, d)=1$, if a direct link exists between $c$ and $d$ is computed as:

$$
C_{D}(c)=\sum_{k=1}^{N} a\left(c, d_{k}\right)
$$

Closeness centrality measures the average number of steps it takes for an individual node/user to reach every other node/user in the network. Therefore closeness centrality measures how close, on average, a person is to other people in the network [2], [4], [13]. Closeness centrality has been used to identify important nodes within social networks [15].

Therefore, in social recommender systems, effective collaborations/linkages can be recommended for users based on computations of strong closeness centralities. Strong closeness centrality of an individual to other nodes/users in the network signifies the importance of that node/user. This paves the way for the generation of innovative collaborative (social) recommendations for other users in the network.

Betweenness centrality is defined as the measurement of the extent to which a node/user can act as an intermediary or broker to other nodes/users. Consequently, the more times that a particular node/user lies on paths that exist between other pairs of nodes in the network, the higher the betweenness centrality is for that node17. Nodes that have a high betweenness centrality may act as brokers between subgroups and they may have stronger membership in surrounding communities [13], [14]

Betweenness centrality is one of the most frequently used centrality measures in research on social networks [13], [14]. Therefore, the computation of high betweenness centrality of nodes/users in a network can enable the innovative social recommendation of nodes/users with stronger memberships within subgroups of the community/network as well as other surrounding communities.

The computation of the betweenness centrality of $a$ and $b$ whereby $g_{a, b}$ is the total number of geodesic paths linking $a$ and $b$ and $g_{a, b}(a)$ is the number of geodesic paths that include $a$ is defined as:

$$
C_{B}(a)=\sum_{j=1}^{N} \sum_{k=1}^{j-1} \frac{g_{a b}(a)}{g_{a b}}
$$

\subsubsection{Communities}

A community is defined as a clustering of entities that are "closely" linked to each other, either through direct linkage or through some easily accessible entities that can act as intermediates [16]. Factors that drive individuals to form communities involve social relationships of various forms and backgrounds, therefore communities naturally reflect social relationships among people [2], [4].

Members of a given community are likely to interact more with members of the same community than interacting with other members of different communities within a population [4]. Because wireless mobile devices are usually carried by people, it is possible to extend the concept of social communities to recommender systems through community detection algorithms [2]

Mobile device users within the same community have higher chances of interacting and socializing with each other. Therefore utilizing the right community detection algorithm will improve and sustain the communities they actually belong to and hence such a scenario can be used to generate a recommended community for a user.

The major challenge for a community structure to be introduced in a social network is the design/development of the community detection algorithm.

The two methods that can be used to form a community are: centralized community detection [17], and distributed community detection [18]. The centralized procedure requires full knowledge of the whole social network and its ties, while in the distributed procedure each node/user is able to detect the community it belongs to.

Betweeness centrality and community-based algorithms have been used by researchers for different network analysis methods such as SCAN [13] and E-Mail as Spectroscopy [14]. Innovatively, different presentation session venues can be successfully recommended to participants at a smart conference based on their research interest relationships through community detection algorithms.

For example, degree centrality and social ties can be incorporated with context to successfully recommend communities (presentation sessions) to participants at a smart conference. In such a method, the first step (Screening) will involve analyzing and monitoring participants within smart conference to ascertain potential levels of social ties and degree centralities, in order to select possible presentation 
sessions. In the second step (Computation), computations of tie strengths between participants screened in the first step are done and stored at each point in time using relevant techniques. Based on strong social tie and degree centrality computations, between presenters and participants (collected in step 2) in accordance to threshold values, the third step (Recommend), uses strong similarity modeling to recommend presentations session venues/sub groups (communities) to participants who make a social recommendation request.

\subsubsection{Social Neighbors/Friendship}

In sociology, the term friendship is another concept that describes close personal relationships. In order for two users/nodes to be considered as friends to each other in a social network, they have to have long-lasting and regular contacts as well as share common interests in the real-world [4].

Furthermore, individuals often befriend others who share/have similar and common interests, frequently meet with each other and perform similar activities. Therefore, through the determination of the contact history and/or common interests between two users, a recommender system can generate an efficient recommendation for either of the users.

By interacting only with others who are similar to ourselves, anything that we experience as a result of our position gets reinforced. Homophily is an important factor/reason of making friends. Homophily is the principle that a contact between similar people occurs at a higher rate than among dissimilar people [19]. The general fact of homophily means that behavioral, genetic, cultural or material information that flows through networks will tend to be localized among people with similar characteristics/feature.

\section{MODELING AND EVALUATING THE PERFORMANCE OF SOCIALLY-AWARE RECOMMENDER SYSTEMS}

The differences, similarities and characteristics involving interactive and non-interactive recommender systems has been presented by Olmo and Gaudioso [20]. Interactive recommender systems do not require interaction data of users to be obtained by an external component/system but by the recommender system itself. However in non-interactive recommenders the user's interaction data can be collected from an external system which is not part of the recommender system at all.

After integrating social properties, it is important to determine the definite type of socially-aware recommenders i.e. whether interactive or non-interactive in order to establish which performance metrics to utilize in experimental evaluation. As mentioned above, socially-aware recommendation deals with modeling and predicting user tastes and preferences through the incorporation of available social information into the recommendation process, represented as explicit additional categories of data. These long-term preferences and tastes are usually expressed as ratings and are modeled as the function of not only items and users, but also of the social information obtained from computation of some social properties as illustrated above.

In other words, ratings are defined with the Rating Function as: $R$ : User $\times$ Item $\times$ Social $\rightarrow$ Rating, where User and Item are the domains of users and items respectively, Rating is the domain of ratings, and Social specifies the social information of the users associated with the recommender application.
Innovatively a socially-aware recommendation approach will involve the addition of context to $R$, which will be defined as: $R:$ User $\times$ Item $\times$ Context $\times$ Social $\rightarrow$ Rating, where context is the contextual information obtained from the users associated with the recommender application. Such a rating function $(R)$ will generate more trustworthy and reliable recommendations for users.

In terms of evaluation, classification metrics such as precision, recall and f-measure are applicable for sociallyaware recommendations that involve the social properties described in this article. This is because such metrics deal with binary data. Precision metrics measures a recommender algorithms capability to display only useful items, while it tries to minimize a blend of them with useless ones. Recall metrics measures the coverage of useful items/resources the recommender algorithm/system can achieve. In other words, recall metrics measures the ability of a recommender system/algorithm to acquire all useful items/resources present in the pool. F-measure is derived from the combination of precision and recall into a single absolute value [20].

Some other applicable performance/evaluation metrics for socially-aware recommender systems include: Receiver Operating Characteristic (ROC), Predictive Accuracy Metrics such as Mean Absolute Error (MAE), Rank Accuracy Metrics and the Single Decision Making Capacity (SDMC) Metrics proposed by Olmo and Gaudioso [20], which considers not only the algorithm filter but also the evaluation of the recommender as a whole.

\section{CONCLUSION}

Socially-aware recommenders systems that are developed through the integration of social properties improve social awareness and generate more trustworthy and reliable recommendations for users. It is important to consider issues such as which particular social properties and component factors are applicable for socially-aware recommenders. To successfully achieve the incorporation of social properties in socially-aware recommender systems and further perform reliable evaluations, a better and innovative compromise should be embarked upon by various researchers. We anticipate that social properties have a vital role to play in the future research of socially-aware recommender systems. In addition to items and users, future research in recommender systems should include social properties of users in order to improve recommendation efficiency and accuracy. Consequently, such an innovation needs to be thoroughly explored.

\section{REFERENCES}

[1] M. Baglioni, F. Geraci, M. Pellegrini, and E. Lastres, Fast exact computation of betweenness Centrality in Social Networks," in Proc. of the IEEE/ACM Int. Conf. on Advances in Social Networks Anal. and Mining (ASONAM), 26-29 Aug. 2012, pp. 450-456.

[2] N. Vastardis and K. Yang, "Mobile Social Networks: Architectures, Social Properties and Key Research Challenges," IEEE Commun. Surveys \& Tutorials, vol. 15, no. 3, pp.1-17, 2013.

[3] M.B. Danah and N. Ellison, "Social Network Sites: Definition, History, and Scholarship," J. of ComputerMediated Commun., 13(1), Article 11, 2007.

[4] Y. Zhu, B. Xu, X. Shi, and Y. Wang, "A Survey of Social-Based Routing in Delay Tolerant Networks: 
Positive and Negative Social Effects," IEEE Commun. Surveys \& Tutorials, vol. 15, no. 1, pp. 387-401, 2013.

[5] Z. Huang, D. Zeng and H. Chen, "A Comparison of Collaborative-Filtering Recommendation Algorithms for E-commerce," IEEE Intell. Syst., vol. 22, no. 5, pp. 6878, Sept.-Oct. 2007.

[6] L. Baltrunas, B. Ludwig, S. Peer and F. Ricci, "Context Relevance Assessment and Exploitation in Mobile Recommender Systems," Personal and Ubiquitous Comput., 16(5), 507-526, 2011.

[7] A. Zanda, S. Eibe, and E. Menasalvas, "SOMAR: A SOcial Mobile Activity Recommender," Expert Syst. with Applicat., 39(9), pp. 8423-8429, 2012.

[8] Y. Wang, J. Hou, Y.W. Tan, and X. Nie, “A Recommendation-based Matchmaking Scheme for Multiple Mobile Social Networks Against Private Data Leakage," Procedia Comput. Sci., 17, pp. 781-788, 2013.

[9] M. Jamali and M. Ester, "A Matrix Factorization Technique with Trust Propagation for Recommendation in Social Networks," in Proc. of the $4^{\text {th }}$ ACM Conf. on Rec. Syst., September 2010, pp. 135-142.

[10] G. Cardone, A. Corradi, L. Foschini and R. Montanari, "Socio-Technical Awareness to Support Recommendation and Efficient Delivery of IMS-Enabled Mobile Services," IEEE Commun. Mag., 50(6), pp. 8290, 2012

[11] A. Zanda, S. Eibe, and E. Menasalvas, "Dynamic Clustering Process to Calculate Affinity Degree of Users as Basis of a Social Network Recommender," in Web Inform. Syst. Eng., pp. 215-225. Springer Berlin Heidelberg, 2013.

[12] E.M. Daly and M. Haahr, "Social Network Analysis for Information Flow in Disconnected Delay-Tolerant MANETs," IEEE Trans. on Mobile Comput., vol. 8, no.5, pp. 606-621, May 2009.
[13] A. Chin and M. Chignell, "Automatic Detection of Cohesive Subgroups Within Social Hypertext: A Heuristic Approach", New Review of Hypermedia and Multimedia, Taylor and Francis, vol. 14, no. 1, pp. 121143, 2008.

[14] J.R. Tyler, D.M. Wilkinson, and B.A. Huberman, "Email as Spectroscopy: Automated Discovery of Community Structure Within Organizations," The Information Society, vol 21, no. 2, pp. 143-153, 2005.

[15] M. Kas, K.M. Carley, and L.R. Carley, "Incremental Closeness Centrality For Dynamically Changing Social Networks," IEEE/ACM Int. Conf. on Advances in Social Networks Anal. and Mining (ASONAM), 2013, 25-28 Aug. 2013, pp. 1250-1258.

[16] S.Y. Chan, P. Hui, and K. Xu, "Community Detection of Time-Varying Mobile Social Networks," in Complex Sci., pp. 1154-1159, Springer Berlin Heidelberg, 2009. 13

[17] S.Y. Chan, P. Hui, and K. Xu, "Bubble Rap: SocialBased Forwarding in Delay-Tolerant Networks," IEEE Trans. on Mobile Comput., vol. 10, no. 11, pp. 15761589, 2011. 14

[18] R. Tan, G. Xing, J. Chen, W.Z. Song, and R. Huang, "Fusion-Based Volcanic Earthquake Detection and Timing in Wireless Sensor Networks," ACM Trans. on Sensor Networks (TOSN), vol. 9, no. 2, Article 17, 2013. 15

[19] M. McPherson, L. Smith-Lovin, and J.M. Cook, "Birds of a Feather: Homophily in Social Networks," Annual Review of Sociology, pp. 415-444, 2001.

[20] F. H. del Olmo and E. Gaudioso, "Evaluation of Recommender Systems: A new Approach," Expert Syst. with Applicat., 35 (3), pp. 790-804, 2008. 